\title{
Effects of continuous positive airway pressure therapy on nocturnal blood pressure fluctuation patterns in patients with obstructive sleep apnea
}

Hajime Kumagai ( $\square$ kumaguy88@hiroshima-u.ac.jp )

Hiroshima University https://orcid.org/0000-0001-9660-7701

Hiroyuki Sawatari

Hiroshima University: Hiroshima Daigaku

Tetsuro Hoshino

Hiroshima University: Hiroshima Daigaku

Noriyuki Konishi

Hiroshima University: Hiroshima Daigaku

Yuka Kiyohara

Hiroshima University: Hiroshima Daigaku

Kengo Kawaguchi

Hiroshima University: Hiroshima Daigaku

Yoko Haseda

Aichi Medical University Hospital: Aichi Ika Daigaku Byoin

Aki Arita

Aichi Medical University Hospital: Aichi lka Daigaku Byoin

Ryujiro Sasanabe

Aichi Medical University Hospital: Aichi lka Daigaku Byoin

Toshiaki Shomi

Hiroshima University: Hiroshima Daigaku

\section{Research Article}

Keywords: blood pressure fluctuation pattern, continuous positive airway pressure, nocturnal blood pressure fluctuations, obstructive sleep apnea, pulse transit time

Posted Date: February 9th, 2022

DOI: https://doi.org/10.21203/rs.3.rs-1333398/v1

License: (a) (i) This work is licensed under a Creative Commons Attribution 4.0 International License.

Read Full License 

Effects of continuous positive airway pressure therapy on nocturnal blood pressure fluctuation patterns in patients with obstructive sleep apnea

Hajime Kumagai (0000-0001-9660-7701) $)^{1,2,3^{*}}$, Hiroyuki Sawatari (0000-0002-1257-2802) $)^{3,4}$, Tetsuro Hoshino (0000-0001-7134-4714) ${ }^{1,3}$, Noriyuki Konishi ${ }^{1,3}$, Yuka Kiyohara ${ }^{1,3}$, Kengo Kawaguchi ${ }^{1}$, Yoko Haseda $^{3}$, Aki Arita ${ }^{3}$, Ryujiro Sasanabe ${ }^{3}$, Toshiaki Shiomi ${ }^{1,3}$

${ }^{1}$ Department of Sleep Medicine, Graduate School of Biomedical and Health Sciences, Hiroshima University, Hiroshima 7348553, Japan

${ }^{2}$ Hiroshima Minato Clinic, Hiroshima 7340014, Japan

${ }^{3}$ Department of Sleep Medicine and Sleep Disorders Center, Aichi Medical University Hospital, Aichi 4801195, Japan

${ }^{4}$ Department of Perioperative and Critical Care Management, Graduate School of Biomedical and Health Sciences, Hiroshima University, Hiroshima 7348553, Japan

*Corresponding author: Hajime Kumagai

1-2-3 Kasumi, Minami-ku, 7348553 Hiroshima, Japan

Email: kumaguy88@hiroshima-u.ac.jp; Fax: +81-82-257-1922; Phone: +81-82-257-1922

ORCID iD: (D) https://orcid.org/0000-0001-9660-7701 


\section{Abstract word count: 227}

\section{Manuscript word count: 2580}

\section{Number of Tables: 2}

\section{Number of Figure: 4}

Acknowledgments: The authors wish to thank the patients who participated in this study and the sleep technicians who manually scored the polysomnography data.

Author Contribution: Conceptualization, Hajime Kumagai and Toshiaki Shiomi; methodology, Hajime Kumagai, Hiroyuki Sawatari. and Toshiaki Shiomi; validation, Hajime Kumagai, Hiroyuki Sawatari, Tetsuro Hoshino, and Toshiaki Shiomi; formal analysis, Hajime Kumagai and Hiroyuki Sawatari; investigation, Hajime Kumagai, Yuka Kiyohara, and Kengo Kawaguchi; resources, Hajime Kumagai; data curation, Hajime Kumagai, Noriyuki Konishi, Yoko Haseda, and Aki Arita; writing-original draft preparation, Hajime Kumagai; writing-review and editing, Hajime Kumagai, Hiroyuki Sawatari, Tetsuro Hoshino and Toshiaki Shiomi; visualization, Hajime Kumagai and Hiroyuki Sawatari; supervision, Hajime Kumagai, Tetsuro Hoshino, Ryujiro Sasanabe, and Toshiaki Shiomi; project administration, Hajime Kumagai. All authors have read and agreed to the published version of the manuscript. 


\section{ABSTRACT}

Purpose: Continuous positive airway pressure (CPAP) therapy is a well-established treatment for obstructive sleep apnea (OSA). In this study, we aimed to assess the effects of CPAP therapy on nocturnal blood pressure (NBP) fluctuations (NBPFs) during rapid eye movement (REM) and non-REM sleep, and the NBPF patterns in patients with OSA.

Methods: This retrospective study included 34 patients with moderate-to-severe OSA who underwent polysomnography using pulse transit time pre-CPAP therapy and 3-6 months post-CPAP therapy. We investigated NBP and the frequency of NBPFs in REM and non-REM sleep, as well as changes in NBPF patterns post-CPAP therapy.

Results: The apnea-hypopnea index (AHI), arousal index, systolic NBP, diastolic NBP, diurnal systolic BP (SBP), diastolic BP (DBP), and frequency of NBPFs in REM and non-REM sleep were significantly reduced post-CPAP therapy (all $\mathrm{p}<0.01$ ). A higher pre-CPAP AHI was associated with a reduction in diurnal SBP, systolic NBP, and NBPFs post-CPAP $(r=0.32, p=0.028 ; r=0.40, p=0.019 ;$ and $r=0.51, p$ $=0.002$; respectively). However, $58.8 \%$ of the patients showed no NBPF pattern change with CPAP therapy.

Conclusion: In REM and non-REM sleep, almost all sleep-related parameters, NBP, and frequency of NBPFs significantly improved post-CPAP therapy, whereas NBPF patterns showed varying changes 3-6 
months post-CPAP therapy. These results suggest that factors other than OSA influence NBPF pattern changes.

Keywords: blood pressure fluctuation pattern; continuous positive airway pressure; nocturnal blood pressure fluctuations; obstructive sleep apnea; pulse transit time 


\section{INTRODUCTION}

The American Academy of Sleep Medicine guidelines recommend continuous positive airway pressure (CPAP) therapy for patients with obstructive sleep apnea (OSA) and excessive daytime sleepiness [1]. Severe OSA is associated with cardiovascular disease (CVD), including hypertension [26]. A dose-response relationship between OSA severity and hypertension has also been demonstrated [7]. Moreover, the duration of CPAP therapy during sleep is related to its effectiveness in terms of sleepiness, daily functioning, hypertension, and CVD [4-6, 8-10].

In patients with severe OSA, nocturnal blood pressure (NBP) undergoes several fluctuations (NBPFs) [11]. OSA severity is also associated with BP fluctuations and sympathetic nerve activity [12]. Non-dipping NBPF patterns are more frequently observed in patients with OSA $[5,10,13]$ and are reportedly a risk factor for hypertension and CVD. NBPF patterns are also likely to affect the prognosis of CVD in such patients $[4-6,10]$. A significant correlation between OSA severity and non-dipping NBPF patterns during non-REM sleep has been reported [14]. To the best of our knowledge, the changes in the frequency of NBPFs and NBPF patterns pre- and post-CPAP therapy in patients with OSA have not yet been investigated. Therefore, we aimed to investigate the changes in NBP, the frequency of NBPFs during rapid eye movement (REM) sleep and non-REM sleep using pulse transit time (PTT), and NBPF patterns post-CPAP therapy in patients with moderate-to-severe OSA. 


\section{METHODS}

\subsection{Study patients}

In this retrospective study, patients with OSA, who received CPAP therapy, underwent PTT measurement and polysomnography simultaneously. Changes in sleep-related parameters and NBPFs before and after CPAP therapy were investigated. We analyzed 34 patients (males, $\mathrm{n}=25 ; 73.5 \%$ ) who underwent polysomnography at the Hiroshima Minato Clinic, were diagnosed with OSA (apneahypopnea index $[\mathrm{AHI}] \geq 20 / \mathrm{h}$ ), and had started CPAP therapy between November 2018 and June 2019. In Japan, patients with an AHI $\geq 20 / \mathrm{h}$ are covered by medical insurance for CPAP therapy. These patients were included in this study. All patients had undergone polysomnography and had been assessed for NBP, frequency of NBPFs, and NBPF patterns using PTT both pre- and post-CPAP therapy (after 3-6 months). Based on the recorded NBPF patterns, patients were categorized as having a dipping or a non-dipping pattern. All data were collected from available medical records.

The exclusion criteria for the patients were as follows: (i) those aged $<20$ years and $>80$ years, (ii) those with a history of treatment using CPAP or an oral appliance, and (iii) those with sleep disorders other than OSA. This study was approved by the Institutional Review Board of the Aichi Medical University Hospital (approval number: 2021-049). Informed consent was obtained from all patients; no patients opted out of the study. 


\subsection{Polysomnography}

All patients had undergone overnight polysomnography using a Somnotouch-RESP

(Somnomedics, Randersacker, Germany). Polysomnography included electroencephalography, electrooculography, electromyography of submental and anterior tibial muscles, electrocardiography, nasal flow, thoracic and abdominal movement with respiratory effort, oxygen saturation, snoring, finger plethysmography, and body position analysis. DOMINO light software v.1.4.0. (Somnomedics, Randersacker, Germany) was used to analyze the recorded data. Polysomnography scoring was performed manually according to the American Academy of Sleep Medicine scoring criteria (version 2.3) by a certified sleep technologist blinded to the patient information. Apnea was scored upon cessation or a drop of airflow signal to $\leq 90 \%$ from the baseline for at least $10 \mathrm{~s}$. Hypopnea was defined as a $\geq 30 \%$ reduction in airflow for at least $10 \mathrm{~s}$ associated with arousal, or $\geq 3 \%$ oxyhemoglobin desaturation. The AHI and arousal index were calculated as the average number of apnea and hypopnea events per hour and the number of arousals per hour of sleep, respectively. The cumulative time percentage with $\mathrm{SpO}_{2}<90 \%$ (CT90) was the cumulative time spent with oxygen saturation $<90 \%$.

\subsection{CPAP therapy}

CPAP therapy was performed using a CPAP device (S9 and AirSense10, ResMed Ltd., Bella Vista NSW, Australia; or DreamStation, Phillips Respironics, Murrysville, PA, USA). At the start of 
CPAP therapy, the physician explained the importance of patient adherence to CPAP usage in detail. The CPAP pressure setting was adjusted according to the condition of the patient, and the device was used in the auto-mode. Daily usage status was confirmed through remote monitoring of the CPAP device (NemLink ${ }^{\circledR}$, Teijin Pharma, Tokyo, Japan; f’Rens ${ }^{\circledR}$, Fukuda Denshi, Tokyo, Japan; and Care Orchestrator ${ }^{\circledR}$, Phillips Japan, Tokyo, Japan). Patients were followed-up at our clinic 1-2 weeks following CPAP therapy to confirm that the CPAP device was being used by them, evaluate the adequacy of the CPAP pressure, and understand the impressions and problems of the patients with the use of CPAP.

Subsequently, subjective symptoms and remotely monitored usage status were confirmed by either faceto-face or telephonic interviews on a monthly basis. No patients dropped out between the period of initiation of CPAP therapy and a second polysomnography with CPAP. Good adherence was defined as a CPAP usage rate of $\geq 70 \% /$ month and an average CPAP usage time of $\geq 4 \mathrm{~h} /$ day.

\subsection{BP measurement}

BP and frequency of NBPFs were calculated with the DOMINO software using PTT, which was performed at the same time as the polysomnography. PTT is a well-established method for measuring BP. Significantly high correlations between BP measurements obtained using oscillometric and PTT-based methods have also been reported, which proved the efficacy of PTT [15-19]. PTT-BP, i.e., BP obtained using PTT, has several advantages over 24-h ambulatory BP monitoring, such as: (i) noninvasive cuff-less 
measurement that does not disturb sleep, (ii) availability of beat-to-beat BP calculation and short-term BPF analysis, and (iii) detailed monitoring during sleep by continuous recording of short-term NBPFs. Therefore, PTT-BP can noninvasively recognize notable NBPFs due to OSA during sleep. PTT was calculated as the time taken for the R-wave on the electrocardiogram and the corresponding pulse wave to reach the finger plethysmograph. Systolic BP (SBP) and diastolic BP (DBP) were automatically estimated using the DOMINO software, based on once initial BP calibration, using the oscillometric method on the upper arm $[17,18,20]$. Diurnal BP was measured by PTT and obtained on an average of $3-4 \mathrm{~h}$ prior to falling asleep and $1-2 \mathrm{~h}$ after waking up. NBPFs associated with respiratory events were defined as respiratory-related NBPFs. NBPF patterns were defined as: (i) dipping ( $\geq 10 \%$ ), (ii) non-dipping (from $\geq 0 \%$ to $<10 \%$ ), (iii) reverse dipping ( $<0 \%$ ), and (iv) extreme dipping ( $\geq 20 \%$ fall from daytime to nighttime). The frequency of NBPFs was defined as the number of times per hour in which SBP increased by $\geq 12 \mathrm{mmHg}$ within 30 s during sleep.

\subsection{Statistical analysis}

All statistical analyses were performed using JMP 16.0.0 software (SAS Institute Japan, Tokyo, Japan). Continuous variables were expressed as mean \pm standard deviation, and categorical variables were expressed as numbers or percentages. To compare the differences in the pre- and post-CPAP conditions with respect to demographic, polysomnographic, and PTT parameters, paired $t$-tests and Wilcoxon rank- 
sum tests were conducted for continuous variables. For univariate regression analysis, we estimated Spearman's rank or Pearson correlation coefficients. All the comparisons were two-tailed, and a p-value $<$ 0.05 was considered statistically significant.

\section{RESULTS}

3.1. Demographics and effects of CPAP therapy on sleep-related parameters

The demographic characteristics and sleep-related parameters are shown in Table 1. The mean age and body mass index of patients with OSA were $50.5 \pm 10.4$ years and $26.5 \pm 5.2 \mathrm{~kg} / \mathrm{m}^{2}$, respectively, and 12 patients were consuming anti-hypertensives. The mean AHI, arousal index, and CT90 were $39.3 \pm$ $18.1 / \mathrm{h}, 22.7 \pm 14.1 / \mathrm{h}$, and $16.1 \pm 27.7 / \mathrm{min}$, respectively. The CPAP usage rate/month and the average CPAP usage time/day were $86.0 \%$ and $4.9 \mathrm{~h}$, respectively, which indicated good adherence to CPAP therapy.

CPAP therapy significantly improved sleep-related parameters, except for the total sleep time, periodic limb movement index, sleep latency, and REM sleep latency. Furthermore, CPAP therapy significantly reduced the AHI and arousal index during REM and non-REM sleep (all $\mathrm{p}<0.0001$ ) (Figure 1A, B).

3.2. Effects of CPAP therapy on NBP and NBPFs 
The SBP and DBP in REM and non-REM sleep were significantly reduced post CPAP therapy (SBP, $p=0.0045$ and $p=0.0067$ DBP, $p=0.0002$ and $p=0.0003$, for REM and non-REM, respectively) (Figure 2A, B). The average diurnal SBP and DBP were also significantly reduced $(p=0.0079$ and $p=$ 0.0022, respectively). The fluctuation ranges for BP pre- and post-CPAP therapy were $6.1 \mathrm{mmHg}$ and 9.0 mmHg of SBP during REM sleep (REM-SBP), $9.2 \mathrm{mmHg}$ and $10.8 \mathrm{mmHg}$ of DBP during REM sleep (REM-DBP), $3.8 \mathrm{mmHg}$ and $9.6 \mathrm{mmHg}$ of SBP during non-REM sleep (NREM-SBP), and $9.3 \mathrm{mmHg}$ and $9.6 \mathrm{mmHg}$ of DBP during non-REM sleep (NREM-DBP). However, the maximum increase in systolic NBP ( $\triangle \mathrm{s}-\mathrm{NBP})$ did not significantly change post-CPAP therapy. The average diurnal heart rate was not reduced post-CPAP therapy $(\mathrm{p}=0.375)$, though the nocturnal heart rate was significantly decreased $(p=0.0012)$ (Table 2). Pre-CPAP therapy, no statistically significant differences were found between patients consuming and not consuming antihypertensive agents for REM-SBP $(140.3 \pm 25.0$ vs. $129.6 \pm 16.7 \mathrm{mmHg}, \mathrm{p}=0.1436$; respectively $)$ REM-DBP $(92.2 \pm 19.6$ vs. $83.4 \pm 11.0 \mathrm{mmHg}, \mathrm{p}=$ 0.1543; respectively), NREM-SBP $(138.6 \pm 22.6$ vs. $126.8 \pm 16.0 \mathrm{mmHg}, \mathrm{p}=0.0851$; respectively $)$, and NREM-DBP $(92.3 \pm 17.9$ vs. $82.0 \pm 10.3 \mathrm{mmHg}, \mathrm{p}=0.1298$; respectively $)$.

The frequency of NBPFs was significantly reduced in REM and non-REM sleep post-CPAP therapy ( $\mathrm{p}=0.009$ and $\mathrm{p}<0.0001$, respectively) (Figure 2A, B). Respiratory-related NBPFs were also significantly reduced post-CPAP therapy $(\mathrm{p}<0.0001)$. The changes in NBPF patterns pre- and post-CPAP therapy are shown in Figure 3. The prevalence of NBPF patterns such as dipping, non-dipping, reverse- 
dipping, and extreme dipping pre- and post-CPAP ranged from $17.6 \%(\mathrm{n}=6)$ to $14.7 \%(\mathrm{n}=5), 73.5 \%$ $(\mathrm{n}=25)$ to $76.5 \%(\mathrm{n}=26), 8.8 \%(\mathrm{n}=3)$ to $2.9 \%(\mathrm{n}=1)$, and $0 \%(\mathrm{n}=0)$ to $5.9 \%(\mathrm{n}=2)$, respectively. The NBPF patterns in $20(58.8 \%)$ patients did not change, and only five among $25(20.0 \%)$ patients with the non-dipping pattern changed to a dipping or extreme-dipping pattern post-CPAP therapy.

3.3. Correlation between sleep-related parameters and nocturnal BP

A higher AHI pre-CPAP therapy was associated with a greater reduction in diurnal and nocturnal SBP and NBPFs post-CPAP $(r=0.32, p=0.028 ; r=0.40, p=0.019 ;$ and $r=0.51, p=0.002$; respectively). In addition, a higher arousal index before CPAP was associated with a greater reduction in NBPFs after CPAP $(r=0.39, p=0.024)$ (Figure 4).

\section{DISCUSSION}

Our study findings revealed that CPAP therapy significantly reduced NBP and the frequency of NBPFs during REM and non-REM sleep in patients with moderate-to-severe OSA. In addition, a higher AHI before CPAP was associated with significantly reduced diurnal and nocturnal SBP and NBPFs postCPAP therapy.

The American Academy of Sleep Medicine guidelines note the importance of CPAP therapy for patients with OSA [1]. Long-term CPAP therapy has also been reported to have antihypertensive effects 
[8, 21-24]. Our results showed that CPAP therapy significantly reduced SBP, DBP, and NBPFs during REM and non-REM sleep. Furthermore, we observed that diurnal SBP and DBP were also significantly reduced. CPAP therapy is considered effective if SBP is reduced by $2 \mathrm{mmHg}$ and DBP by $1 \mathrm{mmHg}$ [25]. We found that CPAP therapy was effective as an antihypertensive therapy, with additional reductions noted in NBPFs during REM and non-REM sleep.

It is crucial to reduce NBP and NBPFs, given the associated risk of cerebrocardiovascular and organ damage $[4,26,27]$. Compared with non-REM sleep, REM sleep is associated with greater sympathetic activity and cardiovascular instability in healthy individuals and those with OSA [28-30]. In our study, CPAP therapy reduced NBP and the frequency of NBPFs in both REM and non-REM sleep. Moreover, CPAP therapy reduced NBPFs, especially respiratory-related NBPFs. The reduction in NBP suggested that CPAP acted by suppressing sympathetic nerve activation.

While CPAP therapy has been shown to be effective against NBPFs from the first night of use [22], our findings suggest that the positive effects on NBPFs continue for 3-6 months after CPAP. High SBP and DBP are known to increase CVD risk [31]. Our findings suggest that with more severe OSA, there is a greater reduction in SBP, DBP, and frequency of NBPFs due to CPAP therapy, which contributes to a reduced risk of CVD. 
OSA is associated with the type of NBPF patterns_- it significantly increases the risk of non-

dipping patterns [5, 13]. Approximately $59 \%$ of patients with OSA have a non-dipping pattern [10]. In

addition, NBPFs and non-dipping patterns correlate with the severity of OSA [32]. A non-dipping pattern

is associated with hypertension and CVD and is reported to influence the overall prognosis due to CVD

$[4-6,10]$. Patients with severe OSA have an increased risk of fatal and non-fatal CVD [33]. In our study, a

non-dipping pattern was observed in $82.4 \%$ and $79.4 \%$ of patients pre- and post-CPAP therapy,

respectively. Although CPAP therapy significantly reduced the AHI as well as NBP and NBPFs, only

$20.0 \%$ of patients changed from a non-dipping pattern to a dipping or extreme-dipping pattern with

CPAP therapy. As OSA increases in severity, the proportion of non-dipping patterns and the risk of CVD

increases, suggesting the need for CPAP therapy to reduce these risks. Determining the cause of the

heterogeneous effects of CPAP therapy on NBPF patterns may reveal whether the risk of hypertension

and CVD and the subsequent prognosis would differ, depending on how NBPF patterns change pre- and

post-CPAP therapy.

CPAP therapy reportedly reduces the risk of developing hypertension and CVD [34] and is

reported to be significantly associated with improved prognosis [35]. Therefore, CPAP should be used

every night to maintain its therapeutic efficacy [8]. Although CPAP adherence is usually considered to be good when CPAP usage is $\geq 70 \% /$ month and the average usage time is $\geq 4 \mathrm{~h} /$ day [36], it has been reported that if CPAP is used only 3-4 h after the onset of sleep, $60-75 \%$ of REM-OSA persists [37]. In 
this study, CPAP therapy improved the AHI, arousal index, NBP, and the frequency of NBPFs in REM and non-REM sleep. CPAP therapy was also effective in controlling diurnal and nocturnal BP. Furthermore, the more severe the OSA, the greater the antihypertensive effect of CPAP therapy. Therefore, long-term and almost daily use of CPAP therapy during both REM and non-REM sleep are important for 24-h BP control and CVD prevention in individuals with greater OSA severity.

This study had some limitations. The sample size of patients was small, and only Japanese patients were included. When considering PTT-BP, clinicians should be aware that the sleeping position, snoring, and arrhythmia affect PTT-BP. Since the BP value used to calculate PTT-BP undergoes only one calibration, it is perhaps better to evaluate BP fluctuations rather than the absolute BP values. In future studies, we hope to increase our sample size and validate our findings in relation to changes in the NBPF patterns and the onset of CVD after long-term CPAP therapy.

In conclusion, nearly all sleep-related parameters, NBP, and frequency of NBPFs significantly improved post-CPAP therapy during REM and non-REM sleep in patients with moderate-to-severe OSA. The varying NBPF patterns post-CPAP therapy suggest that factors other than OSA may influence changes in NBPF patterns. 


\section{References}

[1] Patil SP, Ayappa IA, Caples SM et al (2019) Treatment of adult obstructive sleep apnea with positive airway pressure: An American Academy of Sleep Medicine clinical practice guideline. J Clin Sleep Med 15:335-343. https://doi.org/10.5664/jcsm.7640

[2] Collen J, Lettieri C, Wickwire E, Holley A (2020) Obstructive sleep apnea and cardiovascular disease, a story of confounders! Sleep Breath 24:1299-1313. https://doi.org/10.1007/s11325-019-01945-W

[3] Sawatari H, Chishaki A, Ando SI (2016) The epidemiology of sleep disordered breathing and hypertension in various populations. Curr Hypertens Rev 12:12-17. https://doi.org/10.2174/1573402112666160114093307

[4] Parati G, Ochoa JE, Bilo G. (2012) Blood pressure variability, cardiovascular risk, and risk for renal disease progression. Curr Hypertens Rep 14:421-431. https://doi.org/10.1007/s11906-012-0290-7

[5] Seif F, Patel SR, Walia HK et al (2014) Obstructive sleep apnea and diurnal nondipping hemodynamic indices in patients at increased cardiovascular risk. J Hypertens 32:267-275. https://doi.org/10.1097/HJH.0000000000000011

[6] Sasaki N, Ozono R, Edahiro Y et al (2015) Impact of non-dipping on cardiovascular outcomes in patients with obstructive sleep apnea syndrome. Clin Exp Hypertens 37:449-453. https://doi.org/10.3109/10641963.2015.1057833

[7] Bouloukaki I, Grote L, McNicholas WT et al (2020) Mild obstructive sleep apnea increases hypertension 
risk, challenging traditional severity classification. J Clin Sleep Med 16:889-898.

https://doi.org/10.5664/jcsm.8354

[8] Weaver TE, Maislin G, Dinges DF et al (2007) Relationship between hours of CPAP use and achieving normal levels of sleepiness and daily functioning. Sleep 30:711-719. https://doi.org/10.1093/sleep/30.6.711

[9] Sawyer AM, Gooneratne NS, Marcus CL et al (2011) A systematic review of CPAP adherence across age groups: Clinical and empiric insights for developing CPAP adherence interventions. Sleep Med Rev 15:343-356. https://doi.org/10.1016/j.smrv.2011.01.003

[10] Cuspidi C, Tadic M, Sala C et al (2019) Blood pressure non-dipping and obstructive sleep apnea syndrome: A meta-analysis. J Clin Med 8. https://doi.org/10.3390/jcm8091367

[11] Xu J, Ding N, Zhang X et al (2018) Nocturnal blood pressure fluctuation and associated influential factors in severe obstructive sleep apnea patients with hypertension. Sleep Breath 22:1045-1052. https://doi.org/10.1007/s11325-018-1634-6

[12] Xu J, Ding N, Chen L et al (2019) Inducers of post-apneic blood pressure fluctuation monitored by pulse transfer time measurement in obstructive sleep apnea varied with syndrome severity. Sleep Breath 23:769-776. https://doi.org/10.1007/s11325-018-1770-z

[13] Mokhlesi B, Hagen EW, Finn LAet al (2015) Obstructive sleep apnoea during REM sleep and incident non-dipping of nocturnal blood pressure: a longitudinal analysis of the Wisconsin Sleep Cohort. Thorax 70:1062-1069. https://doi.org/10.1136/thoraxjnl-2015 
[14] BaHammam AS, Alshahrani M, Aleissi SA et al (2021) Blood pressure dipping during REM and non-

REM sleep in patients with moderate to severe obstructive sleep apnea. Sci Rep 11:7990.

\section{https://doi.org/10.1038/s41598-021-87200-3}

[15] Bilo G, Zorzi C, Ochoa Munera JE et al (2015) Validation of the Somnotouch-NIBP noninvasive continuous blood pressure monitor according to the European Society of Hypertension International Protocol revision 2010. Blood pres monit 20:291-294. https://doi.org/10.1097/MBP.0000000000000124

[16] Pitson DJ, Stradling JR (1998) Value of beat-to-beat blood pressure changes, detected by pulse transit time, in the management of the obstructive sleep apnoea/hypopnoea syndrome. Eur Respir J 12:685-692. https://doi.org/10.1183/09031936.98.12030685

[17] Gehring J, Gesche H, Drewniok G et al (2018) Nocturnal blood pressure fluctuations measured by using pulse transit time in patients with severe obstructive sleep apnea syndrome. Sleep Breath 22:337343. https://doi.org/10.1007/s11325-017-1555-9

[18] Patzak A, Mendoza Y, Gesche H, Konermann M (2015) Continuous blood pressure measurement using the pulse transit time: Comparison to intra-arterial measurement. Blood Press 24:217-221. https://doi.org/10.3109/08037051.2015.1030901

[19] Kumagai H, Sawatari H, Hoshino T et al (2020) Nocturnal blood pressure fluctuations in patients with rapid eye movement-related obstructive sleep apnea. J Clin Med 10. https://doi.org/10.3390/jcm10215023 [20] Gesche H, Grosskurth D, Küchler G and Patzak A (2012) Continuous blood pressure measurement by 
using the pulse transit time: comparison to a cuff-based method. Eur J Appl Physiol 112:309-315.

https://doi.org/10.1007/s00421-011-1983-3

[21] Marin JM, Agusti A, Villar I et al (2012) Association between treated and untreated obstructive sleep

apnea and risk of hypertension. JAMA. 307:2169-2176. https://doi.org/10.1001/jama.2012.3418

[22] Picard F, Panagiotidou P, Tammen AB et al (2021) Nocturnal blood pressure and nocturnal blood

pressure fluctuations: the effect of short-term CPAP therapy and their association with the severity of obstructive sleep apnea. J Clin Sleep Med [Ahead of print]. https://doi.org/10.5664/jcsm.9564

[23] Sapiña-Beltrán E, Torres G, Benítez I et al (2019) Differential blood pressure response to continuous positive airway pressure treatment according to the circadian pattern in hypertensive patients with obstructive sleep apnoea. Eur Respir J 54. https://doi.org/10.1183/13993003.00098-2019

[24] Martínez-García MA, Capote F, Campos-Rodríguez F et al (2013) Effect of CPAP on blood pressure in patients with obstructive sleep apnea and resistant hypertension: the HIPARCO randomized clinical trial.

JAMA 310:2407-2415. https://doi.org/10.1001/jama.2013.281250

[25] Patil SP, Ayappa IA, Caples SM et al (2019) Treatment of adult obstructive sleep apnea with positive airway pressure: an American Academy of Sleep Medicine systematic review, meta-analysis, and GRADE assessment. J Clin Sleep Med 15:301-334. https://doi.org/10.5664/jcsm.7638

[26] Misaka T, Niimura Y, Yoshihisa A et al (2020) Clinical impact of sleep-disordered breathing on very short-term blood pressure variability determined by pulse transit time. J Hypertens 38:1703-1711. 
https://doi.org/10.1097/HJH.0000000000002445

[27] Wang C, Zhang J, Liu X et al (2013) Reversed dipper blood-pressure pattern is closely related to severe renal and cardiovascular damage in patients with chronic kidney disease. PLoS One 8:e55419. https://doi.org/10.1371/joural.pone.0055419

[28] Somers VK, Dyken ME, Mark AL, Abboud FM (1993) Sympathetic-nerve activity during sleep in normal subjects. N Engl J Med 328:303-307. https://doi.org/10.1056/NEJM199302043280502

[29] Somers VK, Dyken ME, Clary MP, Abboud FM (1995) Sympathetic neural mechanisms in obstructive sleep apnea. J Clin Investig 96:1897-1904. https://doi.org/10.1172/JCI118235

[30] Findley LJ, Wilhoit SC, Suratt PM (1985) Apnea duration and hypoxemia during REM sleep in patients with obstructive sleep apnea. Chest 87:432-436. https://doi.org/10.1378/chest.87.4.432

[31] Flint AC, Conell C, Ren X et al (2019) Effect of systolic and diastolic blood pressure on cardiovascular outcomes. N Engl J Med 381:243-251. https://doi.org/10.1056/NEJMoa1803180

[32] Marrone O, Bonsignore MR (2018) Blood-pressure variability in patients with obstructive sleep apnea: current perspectives. Nat Sci Sleep 10:229-242. https://doi.org/10.2147/NSS.S148543

[33] Marin JM, Carrizo SJ, Vicente E, Agusti AG (2005) Long-term cardiovascular outcomes in men with obstructive sleep apnoea-hypopnoea with or without treatment with continuous positive airway pressure: an observational study. Lancet 365:1046-1053. https://doi.org/10.1016/S0140-6736(05)71141-7

[34] Barbé F, Durán-Cantolla J, Sánchez-de-la-Torre M et al (2012) Effect of continuous positive airway 
pressure on the incidence of hypertension and cardiovascular events in nonsleepy patients with obstructive sleep apnea: a randomized controlled trial. JAMA 307:2161-2168. https://doi.org/10.1001/jama.2012.4366 [35] Peker Y, Glantz H, Eulenburg C, et al (2016) Effect of positive airway pressure on cardiovascular outcomes in coronary artery disease patients with nonsleepy obstructive sleep apnea. The RICCADSA randomized controlled trial. Am J Respir Crit Care Med 2016;194:613-620. https://doi.org/10.1164/rccm.201601-0088OC

[36] Kribbs NB, Pack AI, Kline LR et al (1993) Objective measurement of patterns of nasal CPAP use by patients with obstructive sleep apnea. Am Rev Respir Dis 147:887-895. https://doi.org/10.1164/ajrccm/147.4.887

[37] Varga AW, Mokhlesi B (2019) REM obstructive sleep apnea: risk for adverse health outcomes and novel treatments. Sleep Breath 23:413-423. https://doi.org/10.1007/s11325-018-1727-2 


\section{Statements and Declarations}

Funding: The authors declare that no funds, grants, or other support were received during the preparation of this manuscript.

Author Contributions: All authors contributed to the study conception and design. Material preparation, data collection and analysis were performed by Hajime Kumagai and Hiroyuki Sawatari. The first draft of the manuscript was written by Hajime Kumagi and all authors commented on previous versions of the manuscript. All authors read and approved the final manuscript.

\section{Compliance with Ethical Standards}

Competing Interests: The authors have no relevant financial or non-financial interests to disclose.

Ethics approval: This study was performed in line with the principles of the Declaration of Helsinki.

Approval was granted by Institutional Review Board of Aichi Medical University Hospital (approval date: July 6, 2021 and protocol code 2021-049)

Consent to participate: Informed consent was obtained from all individual participants included in the study. 


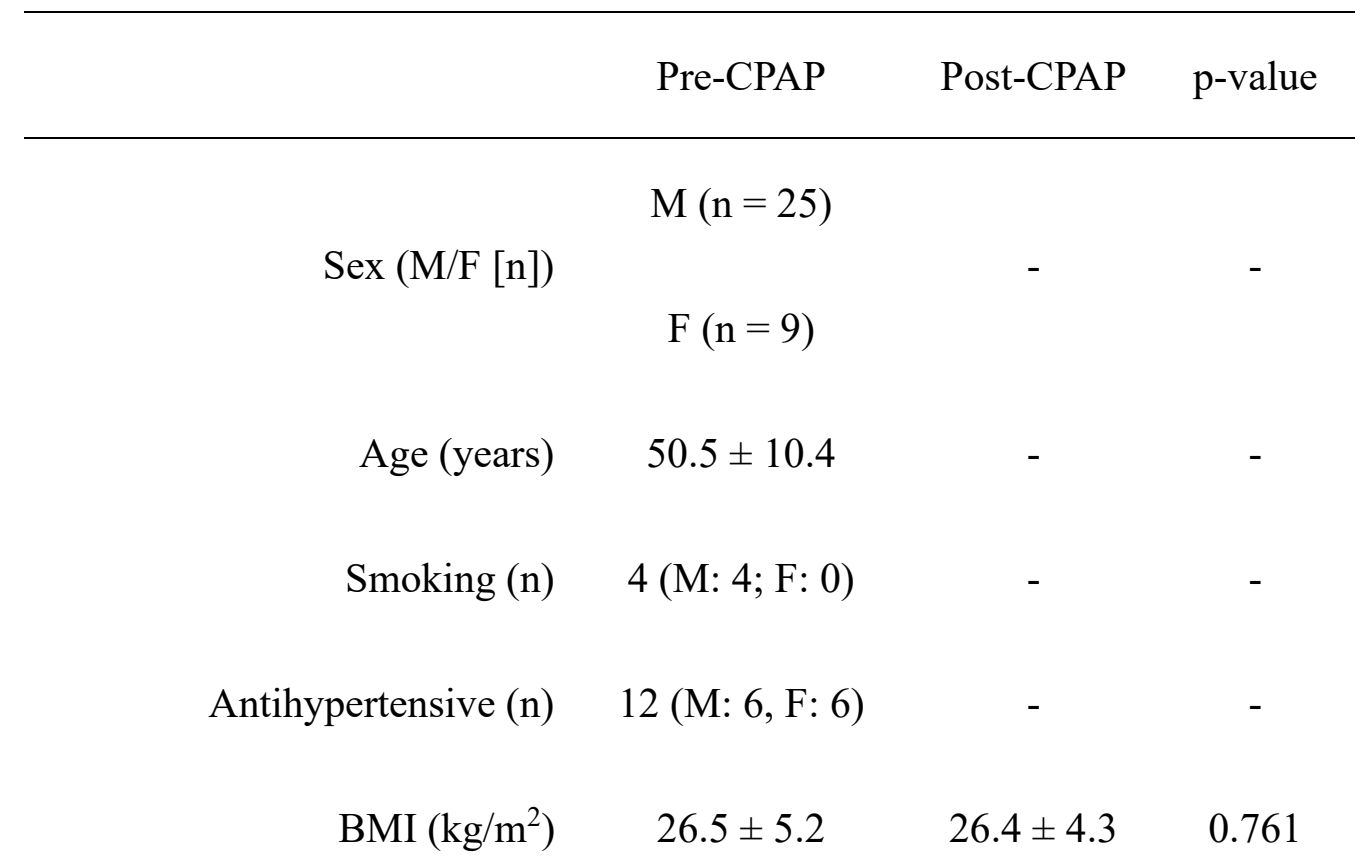

Sleep-related parameters

$\begin{array}{rrrr}\text { TST (min) } & 407.4 \pm 81.2 & 422.0 \pm 72.3 & 0.3042 \\ \text { SE (\%) } & 82.6 \pm 11.8 & 87.0 \pm 7.6 & 0.0077^{*} \\ \text { N1 (\%) } & 29.0 \pm 14.5 & 18.9 \pm 8.4 & <0.0001^{*} \\ \text { N2 (\%) } & 52.5 \pm 13.2 & 56.7 \pm 8.4 & 0.0135^{*} \\ \text { N3 (\%) } & 2.9 \pm 4.6 & 4.0 \pm 4.2 & 0.0152^{*} \\ \text { REM (\%) } & 15.6 \pm 5.1 & 20.4 \pm 5.7 & <0.0001^{*} \\ \text { AHI (/h) } & 39.3 \pm 18.1 & 8.6 \pm 6.1 & <0.0001^{*} \\ & & & \\ \text { REM-AHI (/h) } & 39.3 \pm 18.3 & 6.9 \pm 7.6 & <0.0001^{*} \\ & & & \\ \text { NREM-AHI (/h) } & 39.3 \pm 20.5 & 9.9 \pm 8.3 & <0.0001^{*}\end{array}$




$\begin{array}{rrrr}\text { Arousal index (/h) } & 22.7 \pm 14.1 & 13.3 \pm 6.5 & <0.0001^{*} \\ \text { REM-arousal index (/h) } & 15.9 \pm 14.7 & 6.0 \pm 3.8 & <0.0001^{*} \\ \text { NREM-arousal index (/h) } & 24.4 \pm 14.7 & 15.3 \pm 8.4 & <0.0001^{*} \\ \text { Min SpO (\%) } & 79.5 \pm 8.6 & 86.2 \pm 5.5 & <0.0001^{*} \\ \text { CT90 (min) } & 16.1 \pm 27.7 & 0.9 \pm 1.5 & <0.0001^{*} \\ \text { PLMI (/h) } & 4.1 \pm 8.1 & 4.5 \pm 9.6 & 0.4489 \\ \text { SL (min) } & 20.1 \pm 29.5 & 14.1 \pm 15.7 & 0.2066 \\ & & & \\ \text { REM-SL (min) } & 98.0 \pm 73.0 & 92.7 \pm 60.6 & 0.4455\end{array}$

Continuous variables are expressed as mean \pm standard deviation.

Differences were compared pre- and post-CPAP.

$* \mathrm{P}<0.05$

CPAP, continuous positive airway pressure; BMI, body mass index; TST, total sleep time;

SE, sleep efficiency; N1, sleep stage N1; REM, rapid eye movement; AHI, apnea hypopnea index; NREM-AHI, AHI during NREM sleep; CT90, cumulative time percentage with $\mathrm{SpO}_{2}<90 \%$; PLMI, periodic limb movement index; SL, sleep latency 
Table 2. Changes in nocturnal BP fluctuations, BP, and heart rate

\begin{tabular}{|c|c|c|c|}
\hline & Pre-CPAP & Post-CPAP & p-value \\
\hline Frequency of NBPFs $(/ \mathrm{h})$ & $20.8 \pm 18.2$ & $10.6 \pm 7.5$ & $<0.0001^{*}$ \\
\hline Respiratory-related NBPFs (/h) & $11.4 \pm 13.8$ & $2.3 \pm 2.8$ & $<0.0001 *$ \\
\hline Maximum BP increase $(\mathrm{mmHg})$ & $30.8 \pm 14.3$ & $26.4 \pm 9.8$ & 0.0972 \\
\hline Diurnal average SBP (mmHg) & $138.6 \pm 18.5$ & $131.9 \pm 15.3$ & $0.0079 *$ \\
\hline Diurnal average DBP (mmHg) & $91.8 \pm 13.7$ & $84.1 \pm 10.1$ & $0.0022 *$ \\
\hline Diurnal average HR (bpm) & $80.5 \pm 9.4$ & $80.0 \pm 9.1$ & 0.375 \\
\hline Nocturnal average HR (bpm) & $69.7 \pm 9.3$ & $65.8 \pm 9.1$ & $0.0012 *$ \\
\hline
\end{tabular}

Continuous variables are expressed as mean \pm standard deviation.

Differences were compared pre- and post-CPAP.

$* \mathrm{P}<0.05$

CPAP, continuous positive airway pressure; NBPFs, nocturnal blood pressure fluctuations, SBP, systolic blood pressure; DBP, diastolic blood pressure; HR, heart rate; bpm, beats per minute 


\section{Figure Captions}

Fig. 1 A comparison of sleep related parameters pre- and post-CPAP
(A) REM sleep
(B) non-REM sleep

AHI, apnea hypopnea index; CPAP, continuous positive airway pressure; SD, standard deviation

Gray and white bars indicate the values pre- and post-CPAP, respectively

Values are presented as the mean $\pm \mathrm{SD}$; Error bars represent $\mathrm{SD}$

Fig. 2 A comparison between blood pressures and the NBPFs pre- and post-CPAP
(A) REM sleep
(B) non-REM sleep

CPAP, continuous positive airway pressure; DBP, diastolic blood pressure; NBPFs, nocturnal blood pressure fluctuations; PTT, pulse transit time; SBP, systolic blood pressure; SD, standard deviation

Gray and white bars indicate the values pre- and post-CPAP, respectively

Values are presented as the mean $\pm \mathrm{SD}$; error bars represent $\mathrm{SD}$ 
Fig. 3 Changes in nocturnal blood pressure fluctuation patterns pre- and post-CPAP therapy

CPAP, continuous positive airway pressure

Fig. 4 Correlation between sleep-related and nocturnal blood pressure-related parameters

AHI, apnea hypopnea index; CPAP, continuous positive airway pressure; DBP, diastolic blood pressure; PTT, pulse transit time; SBP, systolic blood pressure

Data availability statement: Data sharing not applicable. 
Figures

Fig 1.

(A) REM

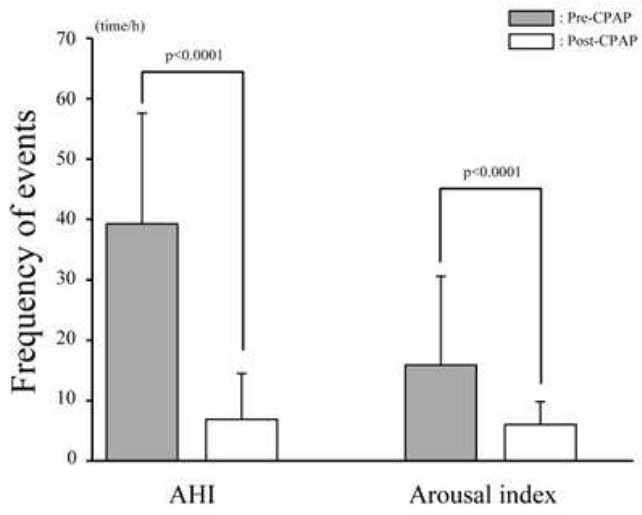

(B) non-REM

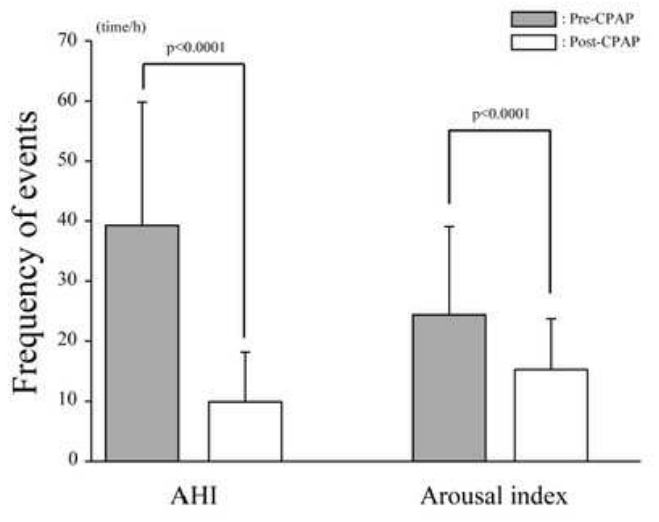

Figure 1

A comparison of sleep related parameters pre- and post-CPAP

(A) REM sleep

(B) non-REM sleep

AHI, apnea hypopnea index; CPAP, continuous positive airway pressure; SD, standard deviation

Gray and white bars indicate the values pre- and post-CPAP, respectively Values are presented as the mean $\pm S D$; Error bars represent SD 
Fig 2.
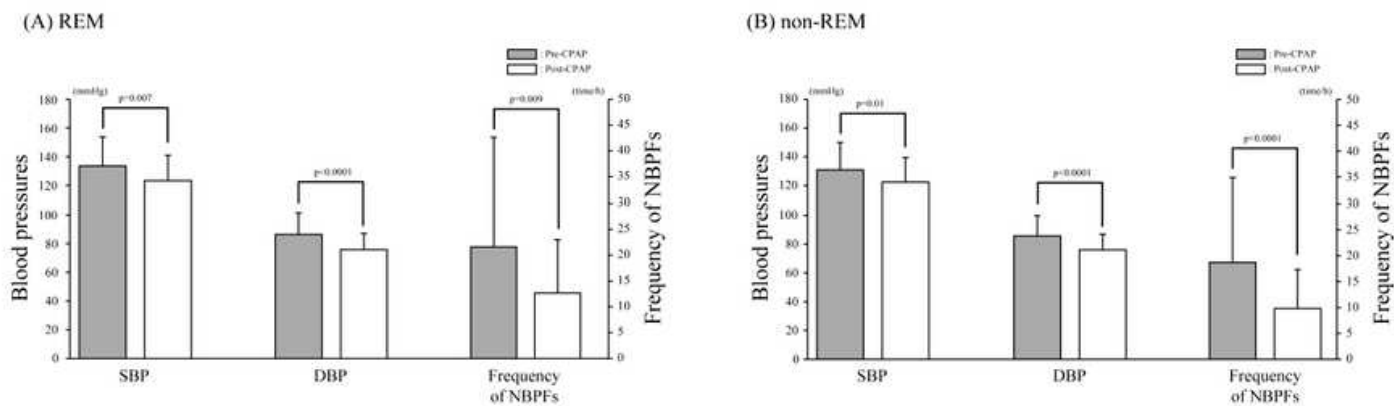

Figure 2

A comparison between blood pressures and the NBPFs pre- and post-CPAP

(A) REM sleep

(B) non-REM sleep

CPAP, continuous positive airway pressure; DBP, diastolic blood pressure; NBPFs, nocturnal blood pressure fluctuations; PTT, pulse transit time; SBP, systolic blood pressure; SD, standard deviation

Gray and white bars indicate the values pre- and post-CPAP, respectively Values are presented as the mean \pm SD; error bars represent SD 
Fig 3.

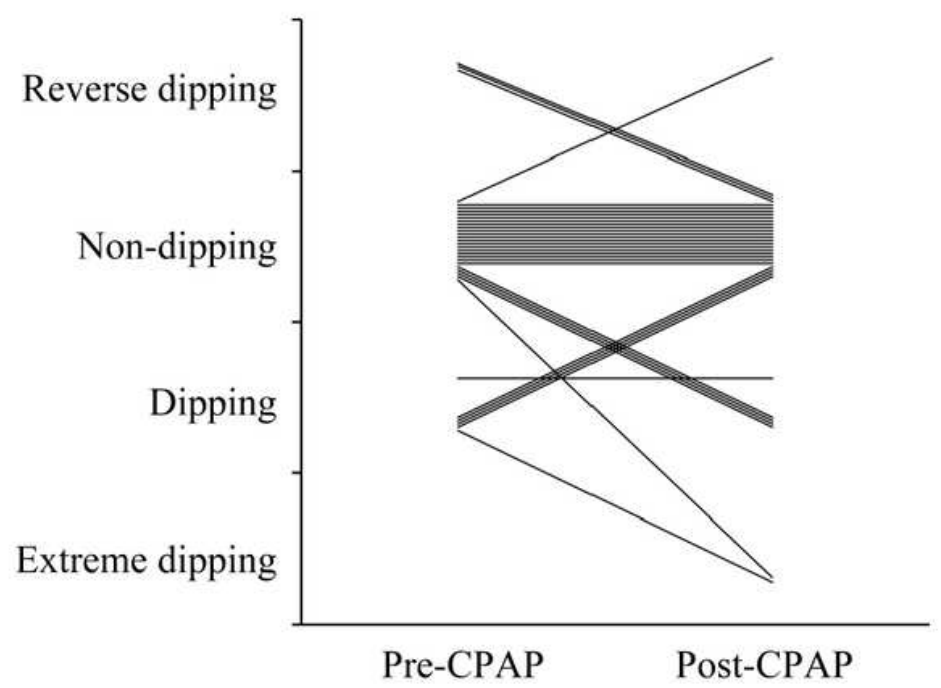

Figure 3

Changes in nocturnal blood pressure fluctuation patterns pre- and post-CPAP therapy CPAP, continuous positive airway pressure 
Fig 4.
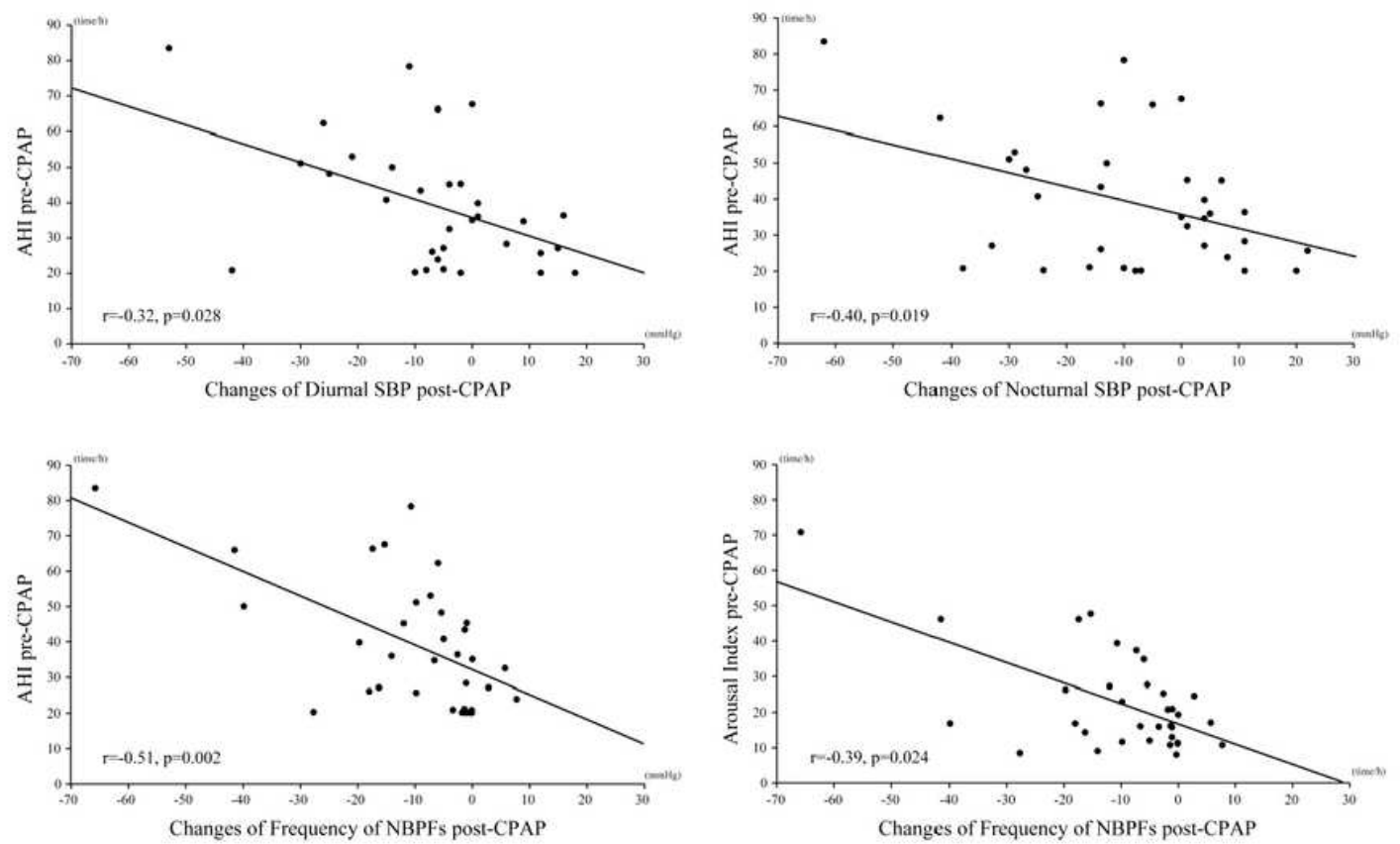

\section{Figure 4}

Correlation between sleep-related and nocturnal blood pressure-related parameters

AHI, apnea hypopnea index; CPAP, continuous positive airway pressure; DBP, diastolic blood pressure; PTT, pulse transit time; SBP, systolic blood pressure 